\title{
The Salience of Colour Terms
}

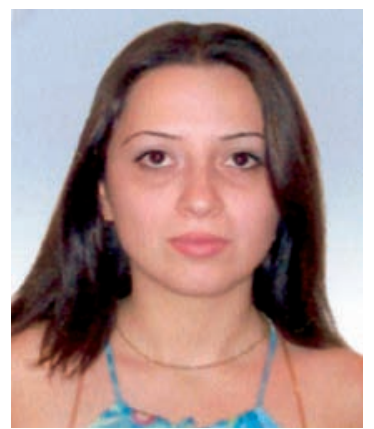

Christine Harutyunyan
$\mathrm{M}$ eaning is one of the most controversial notions in linguistics. At first sight the understanding of this term seems to present no difficulty at all. The term "meaning" is widely used in teaching, theory of translation and so on, but the definition of meaning has always been a matter of interminable discussions.

Culture determines and modifies meanings. It is difficult to pay the necessary attention to the common things that we come across every day. A simple question like "What are the meanings of the words 'red' or 'blue'?" may seem not serious, but at the same time it is very difficult to answer.

The relationship between colour terms and their meanings is not straightforward. The difference in the reference of "red", "orange", "yellow", "green" and "blue" can be described in terms of their variation in hue (the reflection of light at different wavelengths). Physicists recognize two other variables in the analysis of colour: luminosity or brightness (the reflection of more or less light) and saturation (the degree of freedom from dilution with "white"). The range of colour denoted by "black", "grey" and "white" in English differ mainly in respect of luminosity; and there are other common colour terms whose reference must be specified according to all three dimensions of variation: e.g. "brown" refers to a range of colour that is between "red" and "yellow" in hue, of relatively low luminosity and saturation; "pink" is a colour that is reddish in hue, of fairly high luminosity and fairly low saturation. The consideration of these facts might lead us to say that the substance of the field of colour is a three-dimensional (physical or perceptual) continuum. We probably think of colour mainly as hue, but this may not be true of all societies (Lyons, 1968: 431).

Colour terms, though linguistically universal, turn out to be different in different languages from the point of view of their ethnolinguistic salience.

In recent years there has been a good deal of work devoted to the investigation of lexical systems in the vocabularies of different languages, with particular reference to such domains as kinship, colour, flora and fauna, weights and measures, military ranks, moral and aesthetic evolution, various kinds of knowledge, skills and understanding. The results obtained have conclusively demonstrated that the vocabularies of different languages, in certain fields at least, are non-isomorphic, that there are semantic distinctions made in one language which are not made in another, and moreover, that the particular fields may be categorized in a totally different way by different languages.

The language of a particular society is an integral part of its culture, and the lexical distinctions drawn by each language will tend to reflect the culturally important features 
of objects, institutions and activities in the society in which the language operates. As Sapir wrote: "The worlds in which different societies live are distinct worlds, not the same world with different labels attached" (Lyons, 1968: 432 ).

The world is determined by many factors peculiar to the particular nation: these may be history, geography, mode of life, mentality and so on. Any explanation of the connection between linguistic pattern and the rest of culture cannot be abstracted from its ethnographic, social basis. The natural environments in which different societies live, not to mention their institutions and patterns of behaviour, are so diverse, that it is extremely doubtful whether one can talk profitably about semantic structure as an imposition of form upon an underlying (perceptual, physical or conceptual) substance common to all languages. An example of Welsh may be given here to show the differences in colour naming in different languages. Modern Welsh has colour terms corresponding to those of English, yet uses the word "glas" to refer to grass and other growing things even though "glas" otherwise corresponds to English "blue".

For Tarahumara (a language of northern Mexico) speakers, whose language contains only one term for "green" and "blue", the speakers of English exaggerate the psychological distance between colours on the category boundary between "green" and "blue" (Lucy, 1999:183).

The ethnolinguistic differences are accounted for by the specificity of the association of the lexical item with culturally important features of objects in the natural environment.

Each particular language provides names for the thoughts which we want to express. Understanding the meaning of the words requires partly social and partly individual cognition. It is obvious that each person possesses his own individual stereotypes, tastes, superstitions, etc. The same is true for society where different people live.

The psycholinguistic salience of colour terms depends to a great extent on the taste and the sphere of professional interests of the individual.

Investigation has shown that when it is necessary to explain some shade of colour, a person names an object of that colour. Thus, the meaning of the colour term may be understood when it refers to an object the colour of which is perceived similarly by all members of the given society.

It is worth noting in this connection that dictionaries of English frequently define the main colour terms with respect to typical features of the environment.

One hundred informants (students and professors from different faculties, teachers, painters, shop assistants) took part in an experiment which deals with the so-called basic colour terms. According to the results of the experiment colour terms have the following definitions given by the informants. "Blue" is said to refer to the colour of sky (35 inf.), sea (34 inf.), sapphire (10 inf.), turquoise (5 inf.) and screen (16 inf.); "white" to the colour of snow (20 inf.), salt (10 inf.), sugar (10 inf.), lily (8 inf.), wedding gown (17 inf.), egg (13 inf.), angel (7 inf.), milk (15 inf.); "black" refers to the colour of night (25 
inf.), pitch (27 inf.), coal (21 inf.), raven (15 inf.), Negro (12 inf.); "red" refers to the colour of blood (50 inf.), apple (12 inf.), ruby (5 inf.), poppy (13 inf.), currant (4 inf.), tomato (5 inf.), sunset (6 inf.), fire (5 inf.); "green" refers to the colour of grass (36 inf.), leaves (30 inf.), water melon (4 inf.), cucumber (15 inf.), cabbage ( 15 inf.); " yellow" refers to the colour of the sun (23 inf.), melon (10 inf.), lemon (20 inf), gold (20 inf.), butter ( 7 inf.), maize (10 inf.), corn (10 inf.); "grey" refers to the colour of ground (22 inf.), mouse (33 inf.), wolf (18 inf.), hare (14 inf.), sparrow (13 inf.); "brown" refers to the colour of chocolate (45 inf.), cacao (23 inf.), coffee (18 inf.), chestnut (9 inf.), bear (5 inf.); "pink" refers to the colour of rose (69 inf.), lips (16 inf.), face (15 inf.); "violet" refers to the colour of violet (flower) (55 inf.), lilac (34 inf.), iris (11 inf.); "orange" refers to the colour of orange (fruit) (45 inf.), carrot (38 inf.), tangerine (9 inf.), apricot (8 inf.).

Of course, the sun is not exactly of yellow colour, there are not only pink but also red, white and yellow roses, apples besides being red may have green, yellow and even brown colours, but if we come across an expression like "yellow sun", we have no difficulty in understanding it. Similarly, children use yellow pencils to draw the sun. Yellow and red are generally considered to be warm colours, "yellow" being associated with the sun and "red" being associated with fire. This fact can be illustrated by such expressions as "red-hot", "red coals", "fiery red". The Polish expression "czerwony kur" (red cock) is a synonym of the word "fire'.

There is no doubt that we must take into consideration the idiomatic use of the colour terms in different languages, e.g. "white" is "brown" when relating to coffee, "yellow" when referring to wine, and "pink" as applied to people. The huntsmen refer to their bright red jackets as "pink" and we cannot suppose that they are colour-blind.

It is natural for Russians to hear such expressions like: "красное лето", “велая зима", “желтая осень”, “зеленая весна" though none of these seasons are exactly of the mentioned colour. But these expressions are widely spread and very often we come across them in Russian folklore. Yet we hardly meet expressions like "red summer" in English or "पmunupn mưun" in Armenian. It would sound very odd in Russian and Armenian to give a person a surname in colour terms, like "господин Зеленый" or «щumnnG GuGuщ々» but it is quite normal and common to hear a surname in colour terms in English, as in the examples "Mr. Black" or "Mr. Brown".

Thus, the examination of the system of colour terms offers an opportunity to point out the symbolic values of the latter that are common to different language communities. Furthermore, it enables us to investigate the process of the categorization of reality within each ethnic group. 


\section{References:}

1. Lyons J. Introduction to Theoretical Linguistics. Cambridge, 1968.

2. Lucy J. Language Diversity and Thought. Cambridge University Press, 1999.

\section{9กruUuhc funter undten}

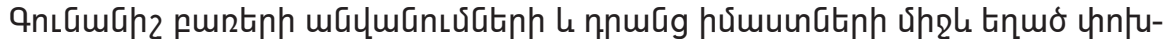

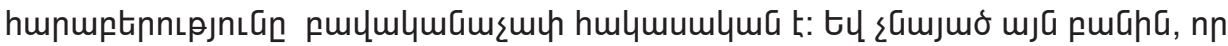

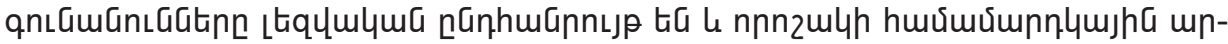

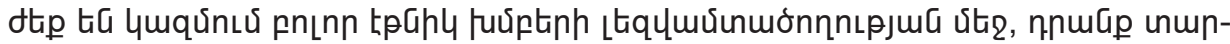

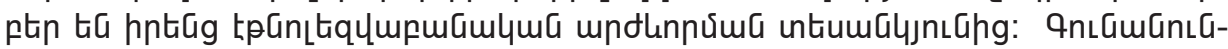

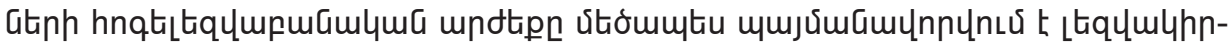

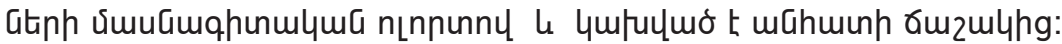

